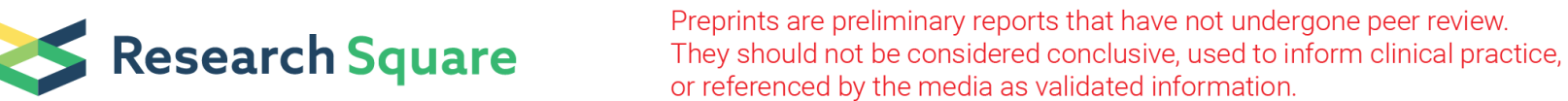

\section{Elevated Plasma Interleukin 34 Levels Correlate with Disease Severity-Reflecting Parameters of Patients with Haemorrhagic Fever with Renal Syndrome}

Kang Tang ( $\nabla$ immu_tangk@163.com )

the Fourth Military Medical University https://orcid.org/0000-0002-6756-0219

Chunmei Zhang

The Fourth Military Medical University

Yusi Zhang

the Fourth Military Medical University

Yun Zhang

the Fourth Military Medical University

Hong Du

the Fourth Military Medical University

Boquan Jin

the Fourth Military Medical University

Ying Ma

the Fourth Military Medical University

Research article

Keywords: Haemorrhagic fever with renal syndrome; cytokine; interleukin 34; clinical parameters

Posted Date: May 23rd, 2019

DOI: https://doi.org/10.21203/rs.2.9746/v1

License: (c) (i) This work is licensed under a Creative Commons Attribution 4.0 International License.

Read Full License

Version of Record: A version of this preprint was published at Infectious Diseases on October 1st, 2019. See the published version at https://doi.org/10.1080/23744235.2019.1672887. 


\section{Abstract}

Background: Haemorrhagic fever with renal syndrome (HFRS) is characterised by an uncontrolled cytokine storm that causes vascular leakage and kidney injury. The cytokine interleukin 34 (IL-34) enhances proliferation and differentiation of myeloid cells and secretion of pro-inflammatory cytokines, which is involved in the pathogenesis of some inflammatory and infectious diseases. However, to date, the role of IL-34 in patients with HFRS is unclear. This study aims to detect the plasma IL-34 levels of HFRS patients and discuss the possible effects of IL-34 in the pathogenesis of HFRS. Methods: IL-34 levels in the plasmas from 52 HFRS patients and 20 healthy controls were quantified using enzymelinked immunosorbent assay. Results: Compared with healthy controls, the plasma IL-34 levels in HFRS patients were significantly elevated at acute phase [37.92 (10.58-73.86) pg/ml vs. 7.13 (3.99-11.31) $\mathrm{pg} / \mathrm{ml}, \mathrm{p}<0.0001]$, and then decreased to the normal levels at convalescent phase. Importantly, IL-34 levels positively correlated with white blood cell count and monocyte count $(r=0.503, p<0.0001$ and $r=$ $0.367, p=0.0003$, respectively), and negatively correlated with platelet count and serum albumin levels ( $r$ $=-0.614, p<0.0001$ and $r=-0.598, p=0.0003$, respectively). Conclusions: IL-34 levels in plasma of HFRS patients were significantly elevated at acute phase and correlated with disease severity-reflecting parameters, which suggests a potential role of IL-34 in the pathogenesis of HFRS and should be future explore.

\section{Background}

Haemorrhagic fever with renal syndrome (HFRS) caused by Hantaan virus (HTNV) is a serious acute infectious disease distributed globally with great harm to human[1]. China is one of the most severe endemic areas of HFRS in the world, and accounts for almost $90 \%$ of human cases globally with the highest incidence each year[2,3]. Patients with HFRS is pathological characterised by increased vascular endothelial permeability, thrombocytopenia and acute kidney injury (AKI)[4]. HTNV infection induced activation of immune system leading uncontrolled cytokine storm, such as dramatically increased cytokines interleukin 1 (IL-1), IL-6, chemokine (C-C motif) ligand 2 (CCL2), CCL4 and tumour necrosis factor- $a(T N F-a)[4,5]$. These cytokines could feedback to enhance the function of immune system against HTNV infection, but also induce the reorganization of the endothelial cytoskeleton and junctions, which mediate an increase in endothelial permeability [6, 7]. Increased endothelial permeability leads to the dysfunction of vascular endothelial barrier, and manifested as petechiae, oedema, and hypotension[8]. The cytokine storm caused dysfunction of vascular endothelial barrier may underlie one of the pathogenesis of HFRS. Additionally, pro-inflammatory cytokines as endogenous pyrogen could induce fever, which is the early clinical manifestation of HFRS[9]. Patients with HFRS showed increased thrombopoiesis and platelet activation, which may induce intravascular coagulation and cause the thrombocytopenia[10,11]. Moreover, the infiltration of inflammatory cells and pro-inflammatory cytokines in kidney tissue could lead to kidney damage[4].

IL-34 is a $39 \mathrm{kDa}$ glycoprotein belonging to the short chain a-helix cytokine family. IL-34 could be secreted by multiple cell types, such as neurons, keratinocytes, synovial fibroblasts, osteoblasts, cytotoxic $T$ cells, 
plasmacytoid dendritic cells, and adipocytes[12-16]. Three kinds of receptors for IL-34 have been identified, including CD115 (colony stimulating factor 1 receptor, CSF-1R; macrophage colony-stimulating factor receptor, M-CSFR), the receptor-type protein-tyrosine phosphatase zeta (PTP- $\zeta$ ) and CD138 (syndecan-1). CD115 is primarily expressed on monocyte precursors, monocytes, dendritic cells and tissue macrophages[17]. Physiologically, expression of IL-34 is restricted to epidermis and central nervous system, which could regulate differentiation of myeloid cells to Langerhans cells and microglia, and modulate their function by binding with $\operatorname{CD} 115[18,19]$. PTP- $\zeta$ is primarily expressed on neural progenitors and glial cells, especially highly expressed in human glioblastomas. The action of IL-34 and PTP- $\zeta$ could inhibit the proliferation, clonogenicity and metastasis of these cancer cells[12, 20]. Furthermore, it is found later that IL-34 could bind to chondroitin sulphate chains of CD138, and modulates IL-34-induced CSF-1R activation and affects cellular migration[21]. Importantly, the expression of IL-34 could be enhanced by pro-inflammatory cytokines TNF- $a$ and IL-1 $\beta$, which is mainly driven by infection and/or inflammation[15, 22, 23], whereas the production of IL-34 could be inhibited by interferon Y (IFN- $\mathrm{Y}$ ) $\square$ bone morphogenetic protein 2 (BMP-2) and transforming growth factor $\beta 1$ (TGF- $\beta 1$ ) [24, 25]. IL34 is involved in inflammatory diseases accompanied by monocytes/macrophages over-proliferation and virus infectious diseases, such as inflammatory bowel disease (IBD)[16], Sjögren's syndrome (SS)[26], hepatitis $C$ virus (HCV)[15] and equine infectious anemia virus (EIAV) infection[27]. These studies showed the potential role of IL-34 as a novel diagnostic and prognostic biomarker of these diseases. Our previous studies have found that blood monocytes were significantly increased in patients with HFRS caused by HTNV infection[28]. However, the change and the role of IL-34 during HTNV infection remains largely unknown. Hence, investigating the changes in the levels of IL-34 and the effect of IL-34 on the pathogenesis of HFRS is an important avenue of study.

In this study, we detected the levels of IL-34 in the plasma of patients with HFRS, and found that IL-34 were significantly increased at the acute phase of HFRS. Importantly, the levels of IL-34 correlated with the typical clinical parameters reflecting disease severity of HFRS, which suggested that IL-34 may play an important role after HTNV infection in patients with HFRS.

\section{Methods}

\section{Study design}

We performed a case-control study. Cases were defined as patients with HFRS. The clinical diagnosis of HFRS was serologically confirmed by detecting specific IgM and IgG antibodies to HTNV. According to the diagnostic criteria from the Prevention and Treatment Strategy of HFRS promulgated by the Ministry of Health, the People's Republic of China, patients with HFRS were classified into four clinical types: mild, moderate, severe, and critical $[29,30]$. (1) Mild: mild kidney damage with proteinuria ranging from " + " to "++" and no obvious oliguric period; (2) moderate: obvious symptoms of effusion (bulbar conjunctiva), uraemia, haemorrhage (skin and mucous membrane), and kidney damage with "+++" urinary protein and occurrence of significant oliguric period; (3) severe: severe effusion (bulbar conjunctiva and either pleura or peritoneum), uraemia, haemorrhage (skin and mucous membrane), and kidney damage with oliguria 
(urine output, $50-500 \mathrm{~mL} /$ day) for $\leq 5$ days or anuria (urine output, $<50 \mathrm{~mL} /$ day) for $\leq 2$ days; (4) critical: $\geq 1$ of the following symptoms during severe disease: visceral haemorrhage, refractory shock, heart failure, pulmonary oedema, brain oedema, severe secondary infection, and severe kidney damage with oliguria (urine output, $50-500 \mathrm{~mL} /$ day) for $>5$ days, anuria (urine output, $<50 \mathrm{~mL} /$ day) for $>2$ days, or a blood urea nitrogen level of $>42.84 \mathrm{mmol} / \mathrm{L}$.

According to the clinical observation, HFRS is defined by five sequential stages: febrile, hypotensive, oliguric, diuretic, and convalescent. These stages are usually classified as the acute phase (febrile, hypotensive, and oliguric stages) and the convalescent phase (diuretic and convalescent stages) [31]. In general, samples were collected at 3-6 days for the febrile or hypotension stage, 7-12 days for the oliguric stage, after 13-18 days for the diuretic stage and after 18 days for the convalescent stage.

Patients with other kidney diseases, diabetes, haematological diseases, cardiovascular diseases, autoimmune diseases, viral hepatitis, and other inflammatory diseases were excluded from this study.

Shaanxi Province, an administrative province in northwestern China, is the most serious HFRS endemic areas of China[2]. Blood samples were intravenously collected from 52 hospitalised HFRS patients between 2015 and 2017 at the Tangdu Hospital of the Fourth Military Medical University (Xi'an, Shaanxi Province, China) and from 20 healthy donors (normal controls, NC). The plasma samples were isolated from EDTA (anticoagulant)-treated blood samples by centrifugation and cryopreserved at $-80^{\circ} \mathrm{C}$ before analysis.

\section{Enzyme-Linked immunosorbent assay for the detection of IL-34 levels in plasma}

The IL-34 levels in plasma were measured using the sandwich enzyme-linked immunosorbent assay (ELISA) kit (R\&D systems, D3400), according to the manual. A monoclonal antibody specific for human IL-34 has been pre-coated onto a microplate. Standards and samples were pipetted into the wells, and any IL-34 present in sample was bound by the immobilized antibody. After washing away any unbound substances, the horse radish peroxidase (HRP)-linked monoclonal antibody for human IL-34 was added to the wells. Then substrate solution Tetramethylbenzidine (TMB) was added to the wells for detection after washing unbound enzyme-linked monoclonal antibody, and stopped by sulfuric acid. Optical densities were determined at $450 \mathrm{~nm}$ with a $570 \mathrm{~nm}$ wavelength correction.

\section{Statistical Analysis}

The statistical analysis was performed in the GraphPad Prism6 software. The comparison between different groups were determined by the Mann-Whitney Utest. The continuous variables were presented as medians with their corresponding interquartile ranges (IQRs). The nonparametric Spearman correlation 
test was used for correlation analysis of IL-34 levels in the plasma with clinical parameters. $p$-values (two-tailed) below 0.05 were considered statistically significant.

\section{Results}

A total of 50 acute-phase plasma and 45 convalescent-phase plasma samples from 52 patients (male/female, 44/8) with HFRS were examined. Meanwhile, 20 plasma samples from 20 healthy donors were assessed in this study. There were 1 mild, 11 moderate, 16 severe, and 24 critical HFRS patients, respectively. The median of age with IQR was 45 (17-82) years for HFRS patients. Furthermore, clinical parameters of all enrolled subjects were summarized in Table 1.

Considering the different phases of HFRS, the IL-34 levels in plasma were 5.3-fold higher at the acute phase compared with the normal controls $[37.92(10.58-73.86) \mathrm{pg} / \mathrm{ml} v \mathrm{vs} .7 .13(3.99-11.31) \mathrm{pg} / \mathrm{ml}, p<$ $0.0001]$, and these levels decreased at the convalescent phase [4.66 (2.63-9.77) pg/ml, $p<0.0001]$ (Fig. 1A). There was no significant difference of IL-34 levels in plasma between HFRS patients at convalescent phase and normal controls $(p>0.05)$. The dynamic changes of plasma IL-34 levels in the same individual also showed the same tendency, that IL-34 levels in plasma were elevated at acute phase and recovered to the normal levels at convalescent phase (Fig. 1B). Between the different HFRS severity groups, there were no significant differences in the plasma IL-34 levels (data not shown).

Further, the relationships were analyzed between plasma IL-34 levels and the clinical parameters detected during hospitalization. Each value of clinical parameters was derived from the same sample. The IL-34 levels in the plasma of HFRS patients positively correlated with white blood cell count (WBC) $(r=0.503, p$ $<0.0001$, Fig. 2A) and monocyte count (MONO) $(r=0.367, p=0.0003$, Fig. 2B), and negatively correlated with the platelet count (PLT) $(r=-0.614, p<0.0001$, Fig. 2C) and serum albumin (ALB) levels $(r=-0.598, p$ $<0.0001$, Fig. 2D).

\section{Discussion}

Our study firstly found that the IL-34 levels in plasma of HFRS patients were significantly elevated at acute phase, and decreased to the normal levels at convalescent phase. Importantly, the IL-34 levels correlated with clinical parameters WBC, MONO, PLT and ALB, which could reflect the severity of the disease, suggesting that IL-34 may play an important role in the pathogenesis of HFRS.

Pro-inflammatory cytokines TNF- $\alpha$ and/or IL-1 $\beta$ stimulation could enhance the IL-34 expression of immune cells such as cytotoxic T cells, plasma cells, monocytes/macrophages and plasmacytoid dendritic cells, and tissue cells such as vascular endothelial cells, fibroblast-like synovial cells, osteoblasts and adipocytes[14, 15,32]. At the acute phase of HFRS patients, levels of TNF-a and IL-1 $\beta$ in serum were significantly elevated [5], which may increase the secretion of IL-34 from above immune cells and tissue cells and contribute to the elevation of plasma IL-34 levels. In our previous study, we found that level of plasma adiponectin, which is predominantly secreted by adipocytes, was significantly 
elevated in HFRS, suggesting that systemic inflammatory response influenced the function of adipocytes in patients with HFRS[33]. The systemic inflammatory response during HTNV infection may also promote the secretion of IL-34 by adipocytes. WBC was increased significantly in HFRS patients. We found that plasma IL-34 levels positively correlated with WBC, which implied that increased WBCs under proinflammatory cytokines stimulation may produce higher level of IL-34 in plasma.

IL-34 is recently described as a pro-inflammatory cytokine by promoting myeloid lineage differentiation, proliferation, and survival and also by inducing secretion of pro-inflammatory cytokines and chemokines such as IL-6, IL-8 and CCL2 in human whole blood[17, 34, 35]. MONO was increased significantly in HFRS patients. We showed that plasma IL-34 levels positively correlated with MONO. Increased IL-34 may act on monocytes and promote their proliferation and survival, leading the accumulation of monocytes in the circulation blood. Importantly, Ciccia et al. have found that increased IL-34 levels of patients with SS were associated with local expansion of $\mathrm{CD} 14^{++} \mathrm{CD} 16^{+}$monocytes, and IL-34 could induce the expansion of both $\mathrm{CD} 14^{++} \mathrm{CD} 16^{-}$and $\mathrm{CD} 14^{++} \mathrm{CD} 16^{+}$monocytes in vitro[36]. We also found that CD $14^{++} \mathrm{CD} 16^{-}$and $\mathrm{CD} 14^{++} \mathrm{CD} 16^{+}$monocytes were remarkably increased in the blood of HFRS patients at the acute phase in our previous study[28]. Therefore, we speculated that the increased plasma IL-34 may contribute to the expansion of blood CD $14^{++} \mathrm{CD} 16^{-}$and $\mathrm{CD} 14^{++} \mathrm{CD} 16^{+}$monocytes in HFRS patients. Interestingly, blocking IL-34 by specific monoclonal antibody could be an effective therapy for myeloid driven inflammatory disease in mice[37], which may provide a new strategy for the therapy of HFRS by inhibit IL-34 induced inflammation.

It has been found that IL-34 could incite the expression of chemokines, such as CCL2 that recruit monocytes into kidney tissues, which aggravates AKI and leads subsequent chronic kidney disease (CKD). The time-related magnitude of macrophage-mediated AKI and subsequent CKD were markedly reduced in IL-34-deficient mice compared with controls. Based on these findings, targeting IL-34 is likely a potential therapeutic strategy to suppress AKI and CKD[38-40]. Dysfunction of glomerular basement membrane during AKI could influence the selective exclusion of ALB from the glomerular filtrate leading to ALB loss in the urine[41]. At the acute phase of HFRS patients, the serum ALB levels were decreased for the injury of kidney function. Here, we found that plasma IL-34 levels negatively correlated with serum ALB levels, suggesting the IL-34 may be involved in the dysfunction of kidney of HFRS patients. Thrombocytopenia induces coagulation disorders and the hemorrhage of HFRS patients. IL-34 levels negatively correlated with PLT, suggesting IL-34 may participated in the decrease of blood platelet during HTNV infection by a complex network.

S2 protein of EIAV specifically induces the expression of IL-34 and may optimize the host macrophage environment to favor viral dissemination and replication, which have a major impact in EIAV induced pathogenesis and disease progression[27]. IL-34 treated macrophages showed slightly higher levels of mitogen-activated protein kinase (MAPK) phosphorylation and associated with higher replication of HIV1[42]. Interestingly, IL-34 treated macrophages could inhibit IFN-y production by NK cells, while IFN- $y$ could inhibit IL-34 expression[14, 15, 24, 25]. IL-34 and IFN- $\gamma$-mediated pathways seemed to be antagonistic. Therefore, IL-34 stimulation may benefit for the replication of viruses. In the acute phase of 
HFRS patients, we found that HTNV load were significantly higher in patients with severer disease severity[43]. Other studies also showed the dysfunction of IFN-y secreting by host immune cells during HTNV infection[44]. The increased plasma IL-34 levels at the acute phase of HFRS patients may contribute to the replication of HTNV and the pathogenesis of HFRS.

\section{Conclusions}

IL-34 could be as a biomarker that help to evaluate inflammatory disease progression and a potential therapeutic target for controlling infections and inflammation. We have found that the IL-34 levels in plasma of patients with HFRS were significantly elevated at acute phase and correlated with clinical parameters reflecting the disease severity, which suggests a potential role of IL-34 in the pathogenesis of HFRS and should be future explore.

\section{Abbreviations}

AKI: acute kidney injury; ALB: serum albumin levels; CKD: chronic kidney disease; CSF-1R: colony stimulating factor 1 receptor; EIAV: equine infectious anemia virus; ELISA: enzyme-linked immunosorbent assay; HFRS: Haemorrhagic fever with renal syndrome; HTNV: Hantaan virus; IBD: inflammatory bowel disease; IL-34: interleukin 34; IQRs: corresponding interquartile ranges; MAPK: mitogen-activated protein kinase; MONO: monocyte count; PLT: platelet count; PTP- $\zeta$ : the receptor-type protein-tyrosine phosphatase zeta; SS: Sjögren's syndrome; TNF-a: tumour necrosis factor-a; WBC: white blood cell count.

\section{Declarations}

\section{Ethics approval and consent to participate}

This study was approved by the Institutional Review Board of the Fourth Military Medical University, and all of the enrolled subjects signed an informed consent form before their blood samples were collected.

\section{Consent for publication}

Not applicable.

\section{Availability of data and materials}

The datasets used and/or analysed during the current study are available from the corresponding author on reasonable request.

\section{Competing interests}


The authors declare that they have no competing interests.

\section{Funding}

This study was supported by the National Natural Science Foundation of China (No. 81871239, No. 81401297 and No. 81501362) and Young Talents Promotion Project of the Association for Science and Technology of Universities and Colleges of Shanxi province in China (No.20170206).

\section{Author contributions}

$\mathrm{KT}$ and $\mathrm{CZ}$ performed the experiments; $\mathrm{YZ}$, Yun-Z and HD provided and organized clinical data; $\mathrm{KT}, \mathrm{CZ}$ and $\mathrm{YM}$ analyzed the data; $\mathrm{KT}, \mathrm{CZ}, \mathrm{BJ}$ and $\mathrm{YM}$ wrote and revised the paper.

\section{Acknowledgments}

We are grateful to all individuals who contributed to this work.

\section{References}

1. Jiang $H$, Zheng $X$, Wang $L$, Du $H$, Wang $P$, Bai $X$. Hantavirus infection: a global zoonotic challenge. Virol Sin.2017; 32(1):32-43.

2. Liang W, Gu X, Li X, Zhang K, Wu K, Pang M, et al. Mapping the epidemic changes and risks of hemorrhagic fever with renal syndrome in Shaanxi Province, China, 2005-2016. Sci Rep.2018; 8(1):749.

3. Tian H, Yu P, Cazelles B, Xu L, Tan H, Yang J, et al. Interannual cycles of Hantaan virus outbreaks at the human-animal interface in Central China are controlled by temperature and rainfall. Proc Natl Acad Sci U S A.2017; 114(30):8041-8046.

4. Jiang H, Du H, Wang LM, Wang PZ, Bai XF. Hemorrhagic Fever with Renal Syndrome: Pathogenesis and Clinical Picture. Front Cell Infect Microbiol.2016; 6:1.

5. Khaiboullina SF, Levis S, Morzunov SP, Martynova EV, Anokhin VA, Gusev OA, et al. Serum Cytokine Profiles Differentiating Hemorrhagic Fever with Renal Syndrome and Hantavirus Pulmonary Syndrome. Front Immunol.2017; 8:567.

6. Vaheri A, Strandin T, Hepojoki J, Sironen T, Henttonen H, Makela S, et al. Uncovering the mysteries of hantavirus infections. Nat Rev Microbiol.2013; 11(8):539-550.

7. Marcos-Ramiro B, Garcia-Weber D, Millan J. TNF-induced endothelial barrier disruption: beyond actin and Rho. Thromb Haemost.2014; 112(6):1088-1102. 
8. Hepojoki J, Vaheri A, Strandin T. The fundamental role of endothelial cells in hantavirus pathogenesis. Front Microbiol.2014; 5:727.

9. Cavaillon JM. Exotoxins and endotoxins: Inducers of inflammatory cytokines. Toxicon.2018; 149:4553.

10. Connolly-Andersen AM, Sundberg E, Ahlm C, Hultdin J, Baudin M, Larsson J, et al. Increased Thrombopoiesis and Platelet Activation in Hantavirus-Infected Patients. The Journal of infectious diseases.2015.

11. Goeijenbier M, Meijers JC, Anfasa F, Roose JM, van de Weg CA, Bakhtiari K, et al. Effect of Puumala hantavirus infection on human umbilical vein endothelial cell hemostatic function: platelet interactions, increased tissue factor expression and fibrinolysis regulator release. Front Microbiol.2015; 6:220.

12. Guillonneau C, Bezie S, Anegon I. Immunoregulatory properties of the cytokine IL-34. Cell Mol Life Sci.2017; 74(14):2569-2586.

13. Ma X, Lin WY, Chen Y, Stawicki S, Mukhyala K, Wu Y, et al. Structural basis for the dual recognition of helical cytokines IL-34 and CSF-1 by CSF-1R. Structure.2012; 20(4):676-687.

14. Chang EJ, Lee SK, Song YS, Jang YJ, Park HS, Hong JP, et al. IL-34 is associated with obesity, chronic inflammation, and insulin resistance. J Clin Endocrinol Metab.2014; 99(7):E1263-1271.

15. Preisser L, Miot C, Le Guillou-Guillemette H, Beaumont E, Foucher ED, Garo E, et al. IL-34 and macrophage colony-stimulating factor are overexpressed in hepatitis $\mathrm{C}$ virus fibrosis and induce profibrotic macrophages that promote collagen synthesis by hepatic stellate cells. Hepatology.2014; 60(6):1879-1890.

16. Camarillo GF, Goyon El, Carballeda JF, Furusho JY. IL-34 and IL-36 family expressing cytotoxic T-cells and plasmacytoid dendritic cells are increased in patients with active inflammatory bowel disease. $J$ Crohns Colitis.2016; 10:S141-S141.

17. Masteller EL, Wong BR. Targeting IL-34 in chronic inflammation. Drug Discov Today.2014; 19(8):12121216.

18. Wang Y, Szretter KJ, Vermi W, Gilfillan S, Rossini C, Cella M, et al. IL-34 is a tissue-restricted ligand of CSF1R required for the development of Langerhans cells and microglia. Nature immunology.2012; 13(8):753-760.

19. Yamaguchi S, Uezato H, Takahashi K. The decrease of IL-34 is the key mechanism to provoke the disappearance of Langerhans cell in the skin lesion of pellagra. J Invest Dermatol.2015; 135:S42-S42.

20. Nandi S, Cioce M, Yeung YG, Nieves E, Tesfa L, Lin H, et al. Receptor-type protein-tyrosine phosphatase zeta is a functional receptor for interleukin-34. The Journal of biological chemistry.2013; 
288(30):21972-21986.

21. Segaliny Al, Brion R, Mortier E, Maillasson M, Cherel M, Jacques $Y$, et al. Syndecan-1 regulates the biological activities of interleukin-34. Biochimica et biophysica acta.2015; 1853(5):1010-1021.

22. Bostrom EA, Lundberg P. The newly discovered cytokine IL-34 is expressed in gingival fibroblasts, shows enhanced expression by pro-inflammatory cytokines, and stimulates osteoclast differentiation. PLoS One.2013; 8(12):e81665.

23. Cheng Y, Yang X, Zhang X, An Z. Analysis of expression levels of IL-17 and IL-34 and influencing factors for prognosis in patients with lupus nephritis. Experimental and therapeutic medicine.2019; 17(3):2279-2283.

24. Chemel M, Brion R, Segaliny Al, Lamora A, Charrier C, Brulin B, et al. Bone Morphogenetic Protein 2 and Transforming Growth Factor beta1 Inhibit the Expression of the Proinflammatory Cytokine IL-34 in Rheumatoid Arthritis Synovial Fibroblasts. Am J Pathol.2017; 187(1):156-162.

25. Wang S, Liu Y, Zhao N, Cui X, Huang M, Li Y, et al. IL-34 Expression Is Reduced in Hashimoto's Thyroiditis and Associated With Thyrocyte Apoptosis. Front Endocrinol (Lausanne).2018; 9:629.

26. Franze E, Monteleone I, Stolfi C, Marafini I, Cupi ML, Caruso R, et al. A Functional Role for II-34 in Sustaining Inflammatory Pathways in Ibd. Digest Liver Dis.2014; 46:S72-S72.

27. Covaleda L, Fuller FJ, Payne SL. EIAV S2 enhances pro-inflammatory cytokine and chemokine response in infected macrophages. Virology.2010; 397(1):217-223.

28. Tang K, Zhang C, Zhang Y, Zhang Y, Zhuang R, Jin B, et al. Elevated Plasma Soluble CD14 Levels Correlate with the Monocyte Response Status During Hantaan Virus Infection in Humans. Viral Immunol.2015; 28(8):442-447.

29. Ma Y, Liu B, Yuan B, Wang J, Yu H, Zhang Y, et al. Sustained high level of serum VEGF at convalescent stage contributes to the renal recovery after HTNV infection in patients with hemorrhagic fever with renal syndrome. Clin Dev Immunol.2012; 2012:812386.

30. Liu Z, Zhao Q, Han Q, Gao M, Zhang N. Serum thrombospondin-1 is altered in patients with hemorrhagic fever with renal syndrome. J Med Virol.2008; 80(10):1799-1803.

31. Zhang Y, Zhang C, Zhuang R, Ma Y, Zhang Y, Yi J, et al. IL-33/ST2 correlates with severity of haemorrhagic fever with renal syndrome and regulates the inflammatory response in Hantaan virusinfected endothelial cells. PLoS Negl Trop Dis.2015; 9(2):e0003514.

32. Nakamichi Y, Mizoguchi T, Arai A, Kobayashi Y, Sato M, Penninger JM, et al. Spleen serves as a reservoir of osteoclast precursors through vitamin D-induced IL-34 expression in osteopetrotic op/op 
mice. Proceedings of the National Academy of Sciences of the United States of America.2012; 109(25):10006-10011.

33. Tang K, Zhang C, Zhang Y, Zhang Y, Zhuang R, Jin B, et al. Sustained High Levels of Both Total and High Molecular Weight Adiponectin in Plasma during the Convalescent Phase of Haemorrhagic Fever with Renal Syndrome Are Associated with Disease Severity. J Immunol Res.2017; 2017:6468097.

34. Lin H, Huang M, Leo C, Ji M, Behrens D, Bosch E, et al. Regulation of myeloid growth and differentiation by a novel cytokine, interleukin-34 (IL-34), via the CSF-1 receptor. Cytokine.2007; 39(1):2424.

35. Lin H, Lee E, Hestir K, Leo C, Huang M, Bosch E, et al. Discovery of a cytokine and its receptor by functional screening of the extracellular proteome. Science.2008; 320(5877):807-811.

36. Ciccia F, Alessandro R, Rodolico V, Guggino G, Raimondo S, Guarnotta C, et al. IL-34 is overexpressed in the inflamed salivary glands of patients with Sjogren's syndrome and is associated with the local expansion of pro-inflammatory CD14(bright)CD16+ monocytes. Rheumatology (Oxford).2013; 52(6):1009-1017.

37. Lin WY, Delarosa D, Young J, Lee D, Jeet S, Huang ZY, et al. Blockade of CSF-1 and IL-34 in mouse model of inflammatory arthritis and colitis show a pathogenic role for IL-34. J Immunol.2013; 190.

38. Baek JH, Zeng R, Weinmann-Menke J, Valerius MT, Wada Y, Ajay AK, et al. IL-34 mediates acute kidney injury and worsens subsequent chronic kidney disease. The Journal of clinical investigation.2015; 125(8):3198-3214.

39. Edwards JK. Acute kidney injury: IL-34 promotes persistent ischaemia-induced AKI. Nat Rev Nephrol.2015; 11(9):504.

40. Weinmann-Menke J, Sollinger D, Schamberger B, Lutz J, Kelley VR. II-34 Mediates Acute Kidney Injury Worsening Subsequent Chronic Kidney Disease. Transpl Int.2015; 28:27-27.

41. Suh JH, Miner JH. The glomerular basement membrane as a barrier to albumin. Nat Rev Nephrol.2013; 9(8):470-477.

42. Chihara T, Suzu S, Hassan R, Chutiwitoonchai N, Hiyoshi M, Motoyoshi K, et al. IL-34 and M-CSF share the receptor Fms but are not identical in biological activity and signal activation. Cell Death Differ.2010; 17(12):1917-1927.

43. Yi J, Xu Z, Zhuang R, Wang J, Zhang Y, Ma Y, et al. Hantaan virus RNA load in patients having hemorrhagic fever with renal syndrome: correlation with disease severity. J Infect Dis.2013; 207(9):14571461. 
44. Wang M, Wang J, Kang Z, Zhao Q, Wang X, Hui L. Kinetics and Immunodominance of Virus-Specific T Cell Responses During Hantaan Virus Infection. Viral Immunol.2015; 28(5):265-271.

\section{Tables}

Table 1: Clinical parameters of HFRS patients in this study.

\begin{tabular}{lll} 
Clinical parameters & Acute phase & Convalescent phase \\
\hline White blood cell count & 15.42 & 8.12 \\
$(\times 109 / \mathrm{L})$ & $(9.61-23.19)$ & $(6.35-10.05)$ \\
\hline Blood platelet count & 44.00 & 196.00 \\
$(\times 109 / \mathrm{L})$ & $(26.50-80.50)$ & $(122.50-278.00)$ \\
\hline Monocyte count & 1.25 & 0.78 \\
$(\times 109 / \mathrm{L})$ & $(0.86-2.24)$ & $(0.53-1.24)$ \\
\hline Blood urea nitrogen level & 15.67 & 12.30 \\
$(\mathrm{mmol} / \mathrm{L} \otimes$ & $(9.10-22.23)$ & $(6.90-18.50)$ \\
\hline Serum creatinine level & 297.50 & 211.90 \\
$(\mu \mathrm{mol} / \mathrm{L})$ & $(128.80-483.60)$ & $(126.40-340.70)$ \\
\hline Serum albumin level & 26.90 & 37.40 \\
$(\mathrm{~g} / \mathrm{L})$ & $(25.60-29.35)$ & $(31.50-41.35)$
\end{tabular}

Values represent medians with the corresponding interquartile range (IQR).

\section{Figures}



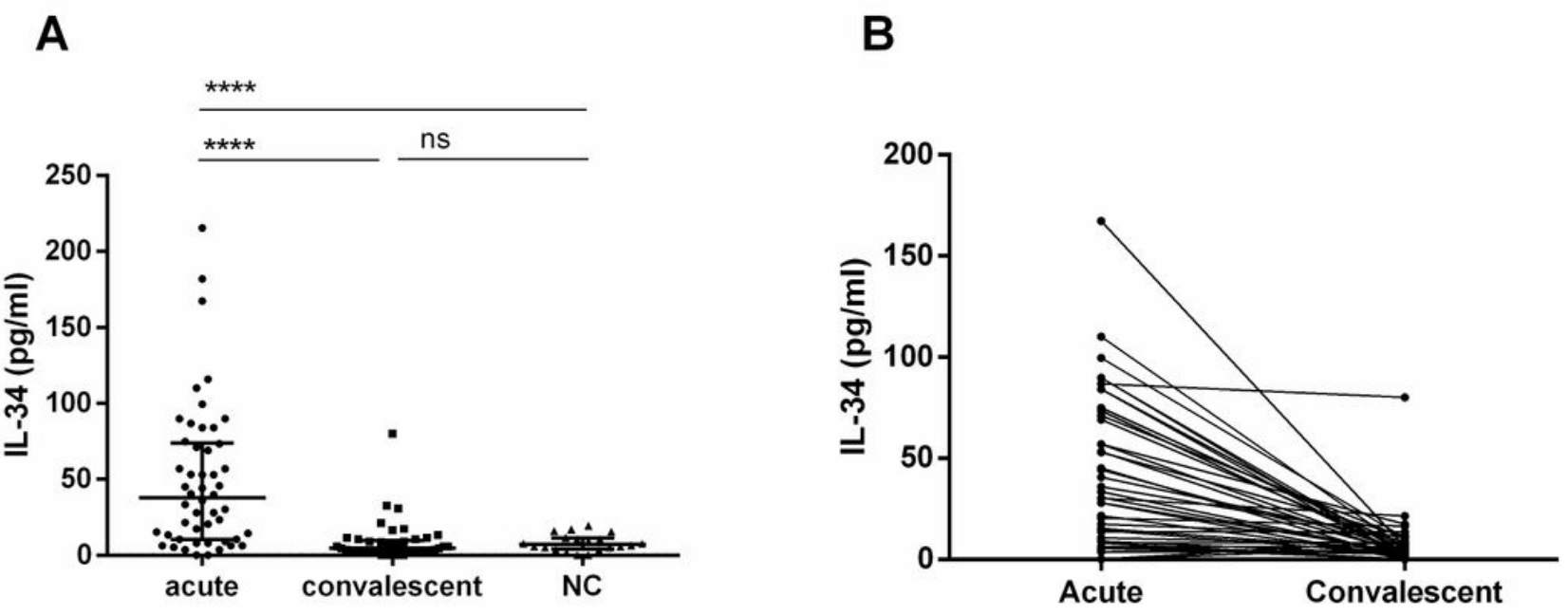

\section{Figure 1}

Dynamic changes of interleukin 34 (IL-34) levels in the plasma of patients with HFRS. (A) A comparison of IL-34 levels in plasma among the acute and convalescent phases of HFRS patients and normal controls (NC). (B) Changes in the levels of IL-34 in the plasma of the same individuals were shown. The Mann-Whitney $U$ test was used to determine the significance of the difference between two groups, and the black lines represent the medians with the corresponding interquartile range. p-values below 0.05 were considered statistically significant. ${ }^{\star \star \star \star} p<0.0001$. 
A

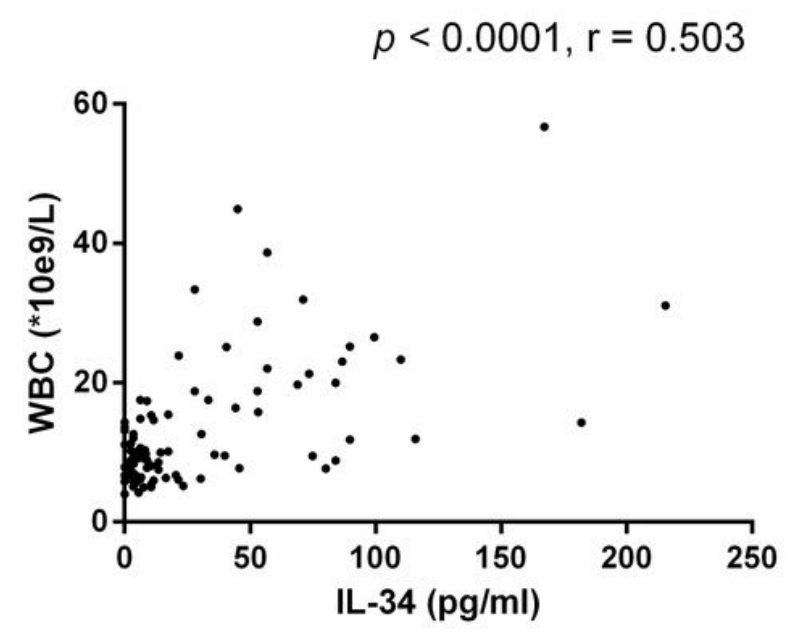

C

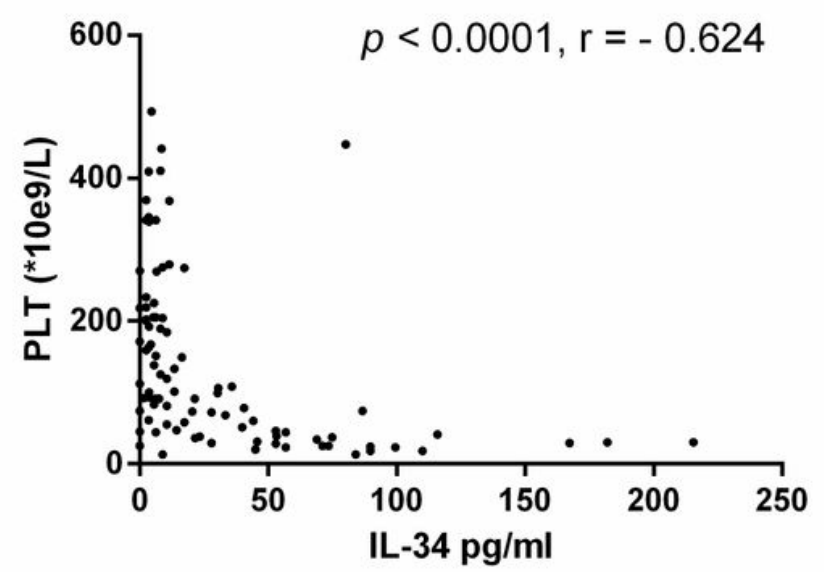

B

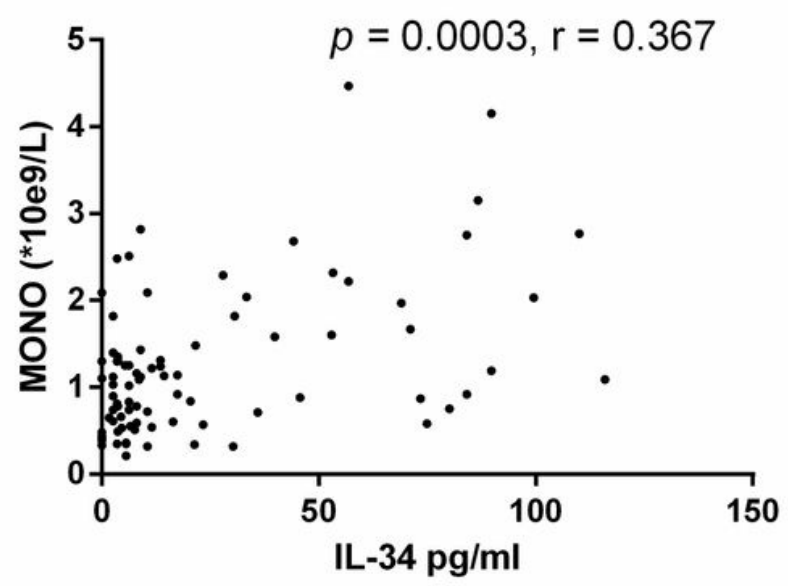

D

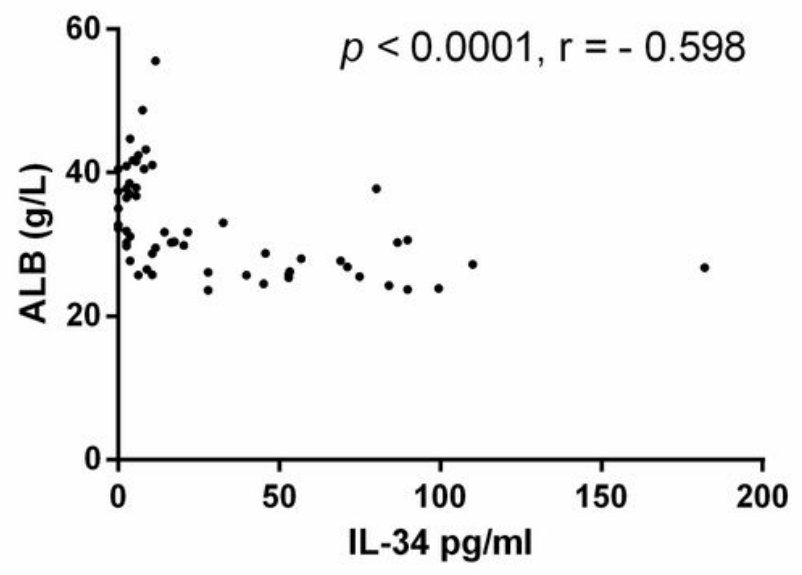

Figure 2

Correlations between the IL-34 levels in plasma and clinical parameters of HFRS patients. Correlation of IL-34 levels in the plasma of HFRS patients with (A) white blood count (WBC), (B) monocyte count (MONO) (C) platelet count (PLT), and (D) serum albumin (ALB) levels were evaluated using the nonparametric Spearman correlation test. Each value of clinical parameters was derived from the same sample. The $r$ denotes the Spearman correlation coefficient. $p$-values below 0.05 were considered statistically significant. 